\title{
Professional gesture in optometry: a case study of video-based field studies
}

\author{
Helena Webb \\ King's College London \\ Department of Management \\ Stamford Street, London, SE1 9NH \\ helena.webb@kcl.ac.uk
}

\author{
Dirk vom Lehn \\ King's College London \\ Department of Management \\ Stamford Street, London, SE1 9NH \\ dirk.vom_lehn@kcl.ac.uk
}

\author{
Christian Heath \\ King's College London \\ Department of Management \\ Stamford Street, London, SE1 9NH \\ christian.heath@kcl.ac.uk
}

\begin{abstract}
The Work, Interaction and Technology Research Centre at King's College London is a centre of excellence in video-based field studies that illuminate the ways in which workplace activities are accomplished through socially organised interactions involving vocal, bodily and material actions. This paper provides a case study of analysis undertaken as part of a recent videobased study of optometry. It describes the processes through which data collection, analysis and dissemination were conducted. In doing so it demonstrates the methodological, conceptual and practical benefits of this kind of approach.
\end{abstract}

Keywords: embodied and multimodal interaction, workplace studies, ethnomethodology, conversation analysis

\section{INTRODUCTION}

The Work, Interaction and Technology Research Centre (WIT) at King's College London is a centre of excellence in the video-based analysis of workplace practice. WIT projects involve the videorecording of naturally occuring activities in settings such as: command and control centres, museums and galleries, markets and auction houses, and healthcare consultations. The resulting data are scrutinised through intricate qualitiative analysis that draws on insights from ethnomethodology (Garfinkel, 1967) and conversation analysis (Sacks, 1992) (EMCA) as well as the emergent field of workplace studies (Luff, Hindmarsh and Heath 2010). This analysis highlights the ways in which workplace activities are accomplished through socially organised interactions involving vocal, bodily and material actions. This includes a commitment to exploring the interactional foundations to technology use, whether these technologies are advanced systems or more mundane artefacts.

This paper provides a case study of a piece of analysis undertaken as part of a recent WIT project. It outlines the processes through which data collection, analysis and dissemination were conducted. In doing so, it demonstrates the value of conducting video-based analysis not only in enabling access to hitherto inaccessible areas of social action, but also in producing a re-usable data corpus with potentially innovative practical applications.

\section{ASSESSING EYE SIGHT AND OCULAR HEALTH}

The "Assessing eye sight and ocular health" project investigated optometry: a form of healthcare practice that provides patients/clients with checks of their vision and eye health (see figure 1). The broad aim of the study was to identify how the tasks of the consultation are achieved, with particular focus on the use of clinical tools and technologies. Data collection took place over a period of 18 months and involved the video recording of consultations in UK optometry practices.

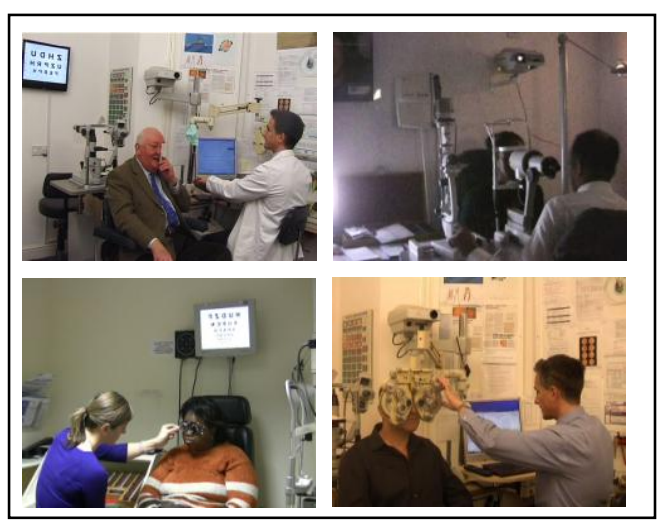

Figure 1: Optometric consultations provide checks of a patient's vision and eye health.

\subsection{Data collection}

Once appropriate NHS and university ethics committee approval had been granted, data collection began with a series of fieldwork observation visits to optometric practices. These visits helped develop a preliminary understanding of the structure of optometric consultations and established the practical requirements for undertaking subsequent video recordings. 
Video recordings were then conducted across seven practices - a mixture of commercial community optometry practices, 'high street' optician chains and student optometry clinics. Participating optometrists and patients were given an information sheet about the project and asked to sign a consent form, indicating their willingness to be recorded and for images from those recordings to be used in dissemination. Before the examination began, a researcher entered the consultation room and switched on a video camera that had been placed in an unobtrusive position, usually in a corner of the room out of the participants' eye-line. The camera then captured the entirety of the consultation and was switched off by the researcher once the room was again empty. This process ensured that consultations proceeded as 'normal', save for the presence of the camera. Over 60 consultations were recorded and the data were then digitised (using software such as Windows Movie Maker, Adobe Premiere Elements etc) to create computer readable video files (avi, wmi etc). These could be easily stored, edited and shared across the research team.

\subsection{Analysis}

Analysis drew on the framework provided by ethnomethodolgy (Garfinkel, 1967) and conversation analysis (CA) (Sacks, 1992), which emphasises the practical accomplishment of social organisation and order through individuals' vocal, bodily and material actions. These actions are themselves socially organised and can be observed to unfold sequentially in interaction. In addition we also drew on related methods and concepts from workplace studies and sociology (e.g., Luff, Hindmarsh and Heath, 2010; Streeck, Goodwin and LeBaron, 2011) that draw attention to embodied and multimodal (i.e., speech, gaze, gesture, manipulation of objects etc) aspects of interaction and explore the systematic interweaving of the material with the social. This approach helps to reveal how tools and technologies gain their sense and significance in and through the interaction amongst participants.

Video data make available for analysis 'small', possibly fleeting actions of speech, gesture etc that can otherwise be easily overlooked but which may nevertheless be key to understanding the accomplishment of social practice and organisation. Further benefits of video lie not only in the opportunity it provides for data to be replayed again and again, but also in the way it enables groups of analysts to work together to observe and discuss data and findings. We made full use of these advantages in the piece of project analysis set out below.

\subsection{Analysing subjective refraction}

As a broadly inductive project, analysis began with:

\subsubsection{The selection of sequences of interest.}

In our project analysis we focused on sequences across the data in which similar types of action occur. In the analysis we describe here, we selected to focus on sequences that occurred during a common test in eye examinations: subjective refraction. As shown in the bottom left image in figure 1, the test involves the patient wearing a trial glasses frame with one eye covered whilst the optometrist brings a lens in front of the uncovered eye and asks questions about any resulting changes in quality of vision. Each eye is tested in turn using a series of lenses and the patient's responses provide information that assists the prescription of any corrective lenses required.

Using video editing software (Windows Movie Maker, Adobe Premiere Elements etc) we made a collection of recorded instances of subjective refraction across the dataset. The video footage always remained the key data but to assist discussion, transcripts of the spoken interaction in these sequences were also produced (figure 2). As is standard in CA, these transcripts include notation markers to indicate potentially relevant phenomena such as word stress, intonation and the length of silences. The transcripts were produced using a combination of word processing programmes and Transana software.

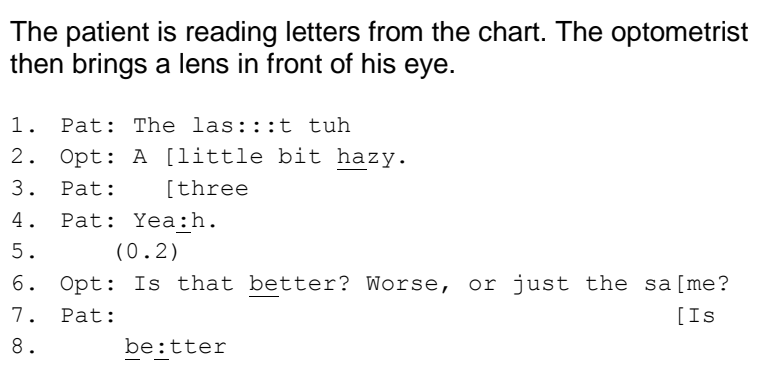

Figure 2: Transcripts of the spoken interaction aid discussion of the video data.

\subsubsection{Case by case analysis}

Analysis then proceeded on a case by case basis. That is, single instances of the sequence of interest were selected and analysed in turn. Analysis began with the 'unmotivated looking' (Sacks 1992), via repeated viewings, at the unfolding of actions in the selected sequence, their design and their consequences for subsequent actions. This process of unmotivated looking was conducted individually and by groups of researchers working together in data sessions, and helped to identify phenomena of particular analytic interest. Discussion of one data clip in a data session led to an interest in the gesture through which the 
optometrist brings the subjective refraction test lens in front of the patient's eye (figure 3 ).
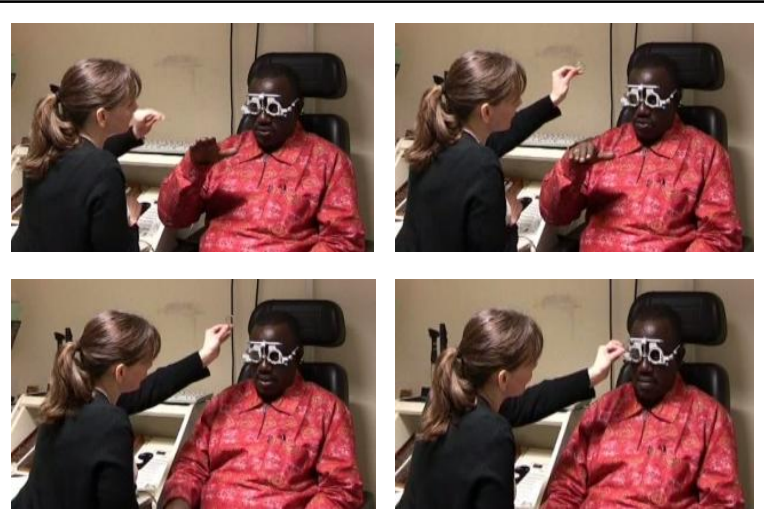

Figure 3: Case by case analysis identifies phenomena of interest - here the gesture through which the optometrist brings the test lens in front of the patient's eye.

It was observed that this gesture had a very distinctive shape, travelling in an arc above the eye and downwards, and was performed in a slow, demonstrative fashion. We then looked across other instances in the data to observe how this gesture was performed by other optometrists in other consultations.

\subsubsection{The creation of a model of the interactional phenomenon}

Looking at cases across the dataset enabled us to build up a model of the interactional phenomenon of interest. We identified several recurrent features of the lens placement gesture. Namely, the lens always travelled a further distance than necessary and came first above the patient's eye before moving downwards. The movement was also slower than other gestures preceding it and had a stylised quality.

Whilst bringing the lens in front of the patient's eye, the optometrist asks some kind of test question to solicit whether the lens changes the patient's quality of vision. We next consolidated our analytic model by carefully scrutinising the design and delivery of these test questions in relation to the lens placement. In order to do this we mapped out the visible and vocal phenomena in our selected data clips (figure 4). This involved charting gaze, body movements etc as they occurred in relation to the transcribed spoken utterances. These data maps were produced using a combination of word processing programmes and video-based transcription software such as CLAN and ELAN. Once again, analysis identified the actions performed in the sequences on a case by case basis before observing similarities across the dataset. Here we observed that the optometrist coupled the test question delivery with the lens placement in a way that consistently ensured the lens arrived shortly before the completion of the question.

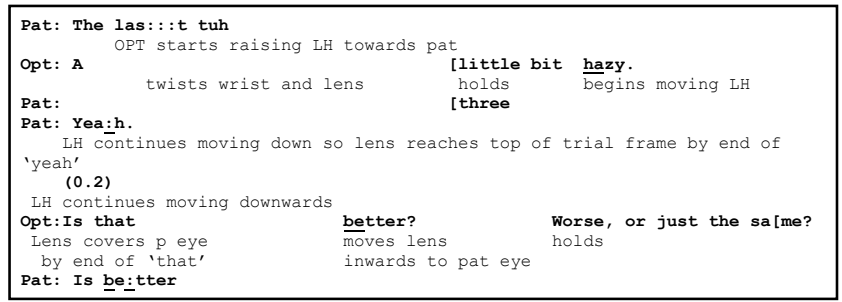

Figure 4: Data maps chart visible actions in relation to the spoken utterances in the sequence.

\subsubsection{Identification of the interactional organisation}

The final stage of the analysis concerned the identification of the interactional organisation at work in the sequences of interest and the relationship of this organisation to the accomplishment of professional tasks in the setting. Once we had built up our empirical model, we discussed our findings in relation to broader understandings of healthcare communication (e.g., Heath, 1986; Heritage and Maynard, 1996) and workplace practice (e.g., Drew, 1992; Luff, Hindmarsh and Heath, 2000). We were also particularly aided by the presence at some of our data sessions of professional optometrists, with whom we were able to discuss technical features of the interaction. Finally, detailed discussion of deviant cases in the model helped us to consolidate our understanding. For instance, in the vast majority of cases in our data, the patient remains still whilst the lens is placed in front of his/her eye. However in one case the patient moves her head away as the lens arrives, perhaps because it is being moved more quickly than usual. The optometrist's subsequent actions repair the placement of the lens and extend the design of the test question. This ensures that the coupling of lens placement and test question is preserved - the lens is in place before the question is complete. The interactional organisation we observe is one in which:

- the placement of the lens attends to clinical features of the test, whilst also displaying to the patient that a test requiring a specific and unusual form of participation is forthcoming. It thereby enables the patient to prepare for the onset of the test.

- the lens placement is accompanied by a test question but gesture and utterance are coupled in a way that ensures the lens is in place before the completion of the question. This accords with a clinical requirement that the patient's eye has time to adjust to the new lens before he/she answers the test question. The patient's eye adjusts to the lens during the completion of the question and this ensures that the patient becomes clinically able to answer the question at the same time he/she becomes interactionally able to do so. This promotes an immediate, 'unthinking' response that 
is preferred in subjective refraction to promote a genuinely instinctive response.

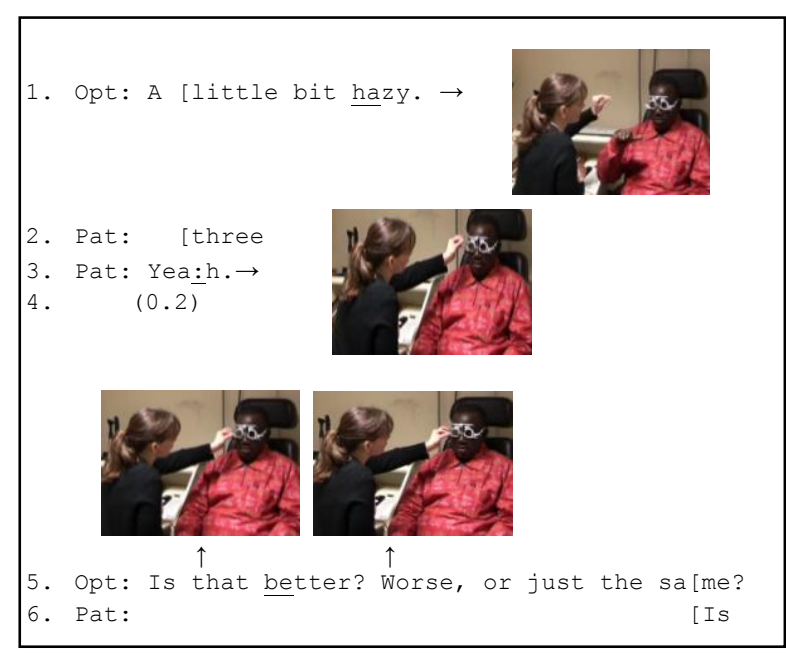

Figure 5: Analysis reveals the interactional organisation at work in the sequence - here, the coupling of the optometrist's visible actions and utterances ensures the lens arrives at the patient's eye before the end of the test question.

The implications of these findings are relevant to conceptual understanding and workplace practice. They reveal the importance of analysing bodily and material actions without regarding them as necessarily subordinate to spoken interaction. We think of the gestures in our data as 'professional gestures': they are both stylised and functional, and alongside spoken utterances they accomplish a workplace task in sophisticated and subtle ways. They are not taught directly in professional training but are nevertheless a prevalent form of professional 'expertise'. A key strand for future research therefore, is to consider how these workplace activities are transformed by the introduction of new technologies. For instance, the subjective refraction test is increasingly conducted using a computerised device known as a phoropter (see figure 1, bottom right image) removing the need for manual lens placement but perhaps involving other forms of professional gesture.

\subsection{Dissemination}

As mentioned above, the video footage always remains the key data in analysis - rather than for instance written summaries or data tables etc. Successful dissemination therefore requires presenting the video in some form. We have particularly benefitted from the permission given by our project participants to display our video footage without image anonymisation in conference presentations and written papers. To disseminate our overall findings we select sequences that illustrate the patterns found across our data and discuss them in detail. During oral presentations data clips can be played, repeated, stopped at key points, slowed down and supplemented by written transcripts. This can be achieved very simply by placing edited clips in PowerPoint presentation files. In written papers, as here, video images of successive moments can be used to demonstrate the trajectory and development of an action (figure 3 ) as well as included in written transcripts (figure 4) to illustrate bodily actions as they relate to spoken utterances. Finally we have found that video data are a key resource in enabling practitioners to examine professional practice within their field. We have successfully used data clips in seminars and conference presentations involving optometric professionals and plan to maximise the practical applications of our project by developing a communication skills portfolio and DVD that draws on our findings.

\section{CONCLUSION}

As this paper has shown, the video-based field studies carried out by the Work, Interaction and Technology Research Centre illustrate how workplace tasks are accomplished through interaction between participants. This interaction involves vocal, bodily and material actions, including the deployment of technologies. The processes of data collection, analysis and dissemination take full advantage of the benefits of video; in particular capturing highly detailed data that can be replayed again and again in order to investigate and illuminate phenomena of conceptual and practical relevance. Consequently, these kinds of study offer topical and methodological contributions to the study of human-computer interaction and related disciplines.

\section{REFERENCES}

Garfinkel, H. (1967) Studies in Ethnomethodology. Englewood Cliffs, N.J.: Prentice Hall.

Drew, P. and Heritage, J. (1992) (Eds.) Talk at Work. Cambridge and New York: Cambridge University Press.

Heath, C. (1986) Body movement and speech in medical interaction. Cambridge: Cambridge University Press.

Heritage, J. and Maynard, D. W. (Eds.) (2006) Communication in medical care: Interaction between primary care physicians and patients. Cambridge: Cambridge University Press.

Goodwin, C.

Luff, P., Hindmarsh, J. and Heath, C. (2000) (Eds.) Workplace studies. New York and Cambridge: Cambridge University Press.

Sacks, H. (1992) Lectures on Conversation Edited by G. Jefferson. Oxford: Basil Blackwell.

Streeck, J., Goodwin, C. and LeBaron, C. 2011. (Eds.) Embodied interaction: Language and body in the material world Cambridge: Cambridge University Press. 